\title{
13 \\ QoS Based Multicast Routing for Multimedia Communications
}

S. Verma, R. K. Pankaj and A. Leon-Garcia

Department of Electrical and Computer Engineering, University of Toronto

10 King's College Road, Toronto, ON M5S 3G4, Canada

Email: (sanjeev, pankaj, alg)@comm.utoronto.ca

Phone: (416) 978- 4766, Fax: (416) 978-4425

\begin{abstract}
Quality of service (QoS) routing is important to support emerging multicast multimedia services. In this paper, we propose a routing metric and use it with two heuristic algorithms to find the multicast tree for guaranteed QoS services. The simulation results shows a marked improvement in network utilizations expressed in terms of cost over other proposed schemes like QOSPF.
\end{abstract}

Keywords: Internet; PGPS; Guaranteed QoS; QOSPF; Multimedia; Multicast Routing.

\section{INTRODUCTION}

In this paper, we consider the problem of setting up the multicast route for a multicast session with guaranteed QoS requirements. The optimum tree in our formulation is a delay bounded minimum steiner tree (Kompella et al. 1993). We assume that we know the delay bound required by the application under consideration.

The paper is organized as follows. In Section 2, we propose the routing metrics to be used with guaranteed QoS applications. In Section 3, we describe two heuristics that can use the routing metric. The performance analysis of the algorithms through simulation is given in Section 4.

\section{METRIC SELECTION FOR GUARANTEED QOS SESSIONS}

Guaranteed QoS applications (Shenker et al. 1996) need firm end-to-end bound on their packet delays and most of these applications will be multicast in nature. Here our goal is to find a multicast route that satisfies the end-to-end delay requirements of the session while having a tree with the minimum cost. In order to achieve our objective, we will first choose an appropriate routing 
metric and then use a heuristic that attempts to optimize that metric. The choice of the metric should reflect the goals that we want to achieve.

We assume that GPS-based scheduling schemes are being used at all the nodes. We use equal allocation scheme (Guerin et al. 1995) to allocate bandwidth at each hop to meet the QoS requirements. If required delay is $d_{\text {reqd }}$ then at each hop $j$, the allocated rate $R_{j}$ is given by

$R_{j}=\max \left[r, \frac{b+(K-1) L}{d_{r e q d}-\sum_{j=1}^{K} \alpha_{j}}\right] \quad \forall j$.

$K$ is the number of nodes and hence $K-1$ is the number of hops in the path. $b$ is the maximum burst size, $L$ is the maximum packet length of the session and $\alpha_{j}$ is the sum of the latency of the scheduler used at node $j$ and propagation delay between node $j-1$ and $j$. If $\widehat{S}=\min _{j} S_{j}$ is the minimum spare capacity or bottleneck bandwidth along the path then $R_{j} \leq \widehat{S}$. As a result, the ability to meet the delay requirement is heavily dependent upon $\widehat{S}$. In addition, Equation 1 indicates that for the same value of $d_{\text {reqd }}$, a larger number of hops, i.e., $K-1$, leads to a higher value of $R_{j}$ and hence more network resources. Hence, to minimize network resource usage while meeting the required delay bound, we should attempt to find routes with large $S$ and small $K$. We propose $\widehat{S} /(K-1)$ as the metric that the routing algorithm attempts to minimize. In the next section, we discuss two heuristic algorithms that use this metric.

We define the following cost function for tree:

$C_{T}=\sum_{j=1} \frac{S_{j}}{S_{j}-R_{j}}$

$R_{j}$ is the actual rate allocated at node $j$ and $S_{j}$ is the spare capacity at node $j$. This cost function will be used to measure the performance of difference algorithms.

\section{$3 \quad$ ROUTING FOR GUARANTEED QOS SERVICES}

\subsection{Shortest Path Algorithm}

The shortest path heuristic finds the shortest path (or best path) from the source to every destination. In our case, we find the path with largest $\widehat{S} /(K-1)$ value for each destination. A tie is broken by choosing a path that has a lower propagation delay. The multicast tree is the union of all paths.

This heuristic has been proposed for different metric by Wang (Wang et 

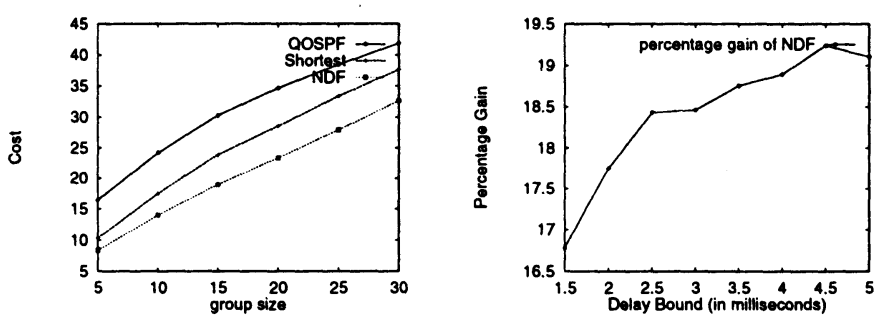

Figure 1 (a)Cost of the tree versus Group size:Delay Bound $=3$ msec,(b)Percentage gain of NDF over Shortest path algorithm as delay bound varies:Group Size $=20$

al. 1996) and is similar to QOSPF (Shenker et al. 1996) being considered by IETF.

\subsection{Heuristics for the Constrained Steiner Tree}

This heuristics was proposed by Takahashi (Takahashi et al. 1980) to find steiner tree and is known as nearest destination first(NDF). Initially we choose the destination for which we can find the largest $\widehat{S} /(K-1)$ path from the source node. Then at each step we find the next nearest destination from the partially constructed tree using the same metric. It is repeated till all the destination nodes are included in the tree. A check is also made at every step to ensure that delay bound violation does not occur.

\section{SIMULATION RESULTS}

We used random euclidean graph for simulation. The spare capacity is uniformly distributed between $20 \mathrm{Mbps}$ and $120 \mathrm{Mbps}$. The maximum burst size was assumed to be 1000 bytes and the maximum length of the session under consideration is assumed to be 100 bytes. The link capacity is assumed to be $200 \mathrm{Mbps}$ and the end-to-end delay bound of $3 \mathrm{msec}$ was assumed for each source destination pair. We randomly generated ten different graphs containing 50 routers or switches. Nodes in the multicast group were randomly chosen.

The simulations were run with the new routing metric introduced in this paper for both shortest path and nearest destination heuristic algorithms. Results were compared with those obtained for QOSPF routing scheme.

The results of the simulation is being summarized in the Figure 1 (a). The results show that both shortest path and NDF using the proposed metric reduce cost compared to QOSPF. For a group size of 5, the cost reduction is $35 \%$ with shortest path and $50 \%$ with NDF. Finally, Figure $1 \mathrm{~b}$ shows that, as 
the desired delay bound becomes loose, the NDF shows better performance over the shortest path algorithm. However, at tighter delay bound there is not much improvement since the increase in cost due to tighter delay bound overshadows the advantages due to path sharing.

\section{CONCLUSIONS}

In this paper, we proposed a new routing metric for guaranteed GQoS applications. We used the metric to find the multicast tree using shortest path and nearest destination first (NDF). The simulation results show that our metric performs better than those used by QOSPF in terms of network utilization. Furthermore, NDF heuristic performs better than shortest path heuristic.

\section{REFERENCES}

Takahashi, H. and Matsuyama, A. (1980) An approximate solution for the steiner tree problem in graphs. Mathematica Japonica, 6, 573-577.

Wang, Z. and Crowcroft, J. (1996) Quality-of-Service Routing for Supporting Multimedia Applications. IEEE J. Select. Areas Commun.,14,12281234

Goyal, P. and Vin, H.M. (1995) Generalized Guaranteed Rate Scheduling Algorithms:A Framework. Technical Report TR 95-30, Department of Computer Sciences, University of Texas Austin

Shenker, S. Patridge, C. and Guerin, R. (1996) Specification of Guaranteed Quality of Service:Internet Draft. http://ietf.org/ids.by.wg/intserv.html

Zhang, Sanchez, Salkewicz, and Crawley (1996) Quality Of Service Path First Routing (QOSPF):Internet Draft http://www.es.net/pub/internetdrafts

Rajagopalan, B. and Nair, R. (1996) Quality of Service (QOS) Based Routing in the Internet- Some Issues: Internet Draft http://ietf.org/ids.by.wg/none.html

Parekh, A.K. and Gallager, R.G. (1994) A Generalized Processor Sharing Approach to Flow Control in Integrated Services Networks: The Multiple Node case. IEEE Trans. Networking,2,135-150

Kompella, V.P. Pasquale, J.C and Polyzos,G.C. (1993) Multimedia Routing for Multimedia Applications. IEEE/ACM Transactions on Networking, 1

Birman, A. Firoiu, V. Guerin, R. and Kandular, D. (1995) Provisioning of RSVP-based services over a Large ATM Network. IBM Research Report- $R C 20250$ 